\title{
EFECTOS TERMODINÁMICOS EN EL DISEÑO DE MATERIALES MULTIMEDIA
}

\author{
(THERMODYNAMICS EFFECTS IN THE MULTIMEDIA MATERIALS DESIGN)
}

\author{
Luis Rodolfo Lara \\ Universidad Nacional de Catamarca (Argentina)
}

\section{RESUMEN}

Este artículo, desde una perspectiva teórica transdisciplinaria, introduce el término entropía en el proceso de diseño y desarrollo de materiales interactivos, de esta manera, se propone el concepto de Sistemas Interactivos Multimedia (SIM) educativos, como un sistema multimedia abierto, volátil y modificable, por tanto, adaptable al entorno con que interactúa.

El sistema presenta modificaciones en cuanto a su estructura cuando interactúa con el alumno, produciendo un proceso de realimentación continua; mediante un análisis cualitativo del comportamiento de la entropía y de la cantidad de información, es posible dar pautas de diseño y también realizar un estudio más exhaustivo de los desplazamientos relativos que presentan.

Así, se orienta el proceso de diseño de estas aplicaciones como un verdadero sistema complejo aceptando, desde una perspectiva constructivista, que el alumno no es un simple usuario de un programa informático, sino que toma un rol protagónico en el proceso de aprendizaje.

Palabras clave: SIM, multimedia, interactividad, sistemas, educación, computación.

\begin{abstract}
This paper, from a transdisciplinary theoretical perspective, introduces the term entropy in the design process and development of interactive materials, this way, it proposes the concept of educational Interactive Multimedia Systems (IMS), as a open, volatile and modifiable multimedia system, therefore, adaptive to the environment with which interact.

The system presents modifications as for its structure when interact with the student, producing a process of continuous feedback; by means of a qualitative analysis of the behaviour of the entropy and of the quantity of information, it is possible to give design rules and also to carry out a more exhaustive study of the relative displacements that they present.
\end{abstract}


This way, it is guided the process of design of these applications like a complex true system accepting, from a constructivist perspective that the student is not a simple user of a computer program, but rather he takes the main role in the learning process.

Keywords: IMS, multimedia, interactivity, systems, computation, education.

Los materiales multimedia interactivos tienen un rol fundamental en educación a distancia, ya que, como todos los materiales instructivos, son portadores de contenidos, tienen una propuesta de acción y constituyen elementos de gran valor didáctico, dado que reemplazan en gran medida la ausencia del profesor, y generan las condiciones necesarias para que el estudiante pueda construir sus conocimientos, autoevaluarse y ser evaluado y orientado por el tutor (Padula Perkins, 2002).

Por otro lado, desde la aparición de los primeros ordenadores, las aplicaciones informáticas y el empleo de los servicios que ofrece Internet, surgen diversos estudios y corrientes de investigación, donde se analizan las potencialidades que pueden ofrecer en el ámbito educativo, sobre todo como recurso de apoyo en educación a distancia, en donde el concepto de interactividad toma mayor relevancia aún. En estos últimos tiempos, es común utilizar como medio tecnológico a los materiales multimedia que sirven de soporte de contenidos para la modalidad no presencial.

El carácter transdisciplinario de este trabajo se basa, justamente en la estructura compleja que presentan estos programas interactivos, como expresa Morin (1999, p. 15) "los desarrollos disciplinarios de las ciencias no sólo aportaron las ventajas de la división del trabajo, también aportaron los inconvenientes de la superespecialización, del enclaustramiento y de la fragmentación del saber. No produjeron solamente conocimiento y elucidación, también produjeron ignorancia y ceguera". Por consiguiente, incorporando principios y conceptos teóricos aparentemente lejanos, que provienen de diversas disciplinas y teorías como la física, la química, la teoría de los sistemas, entre otras, se busca abrir una nueva perspectiva para el diseño de materiales educativos interactivos partiendo de un modelo complejo.

Bajo este nuevo fundamento teórico, un Sistema Interactivo Multimedia (SIM) educativo puede definirse como aquel capaz de poder presentar información, contenidos diversos y actividades en forma textual, sonora y visual de modo coordinada e integrada. Presentación que se realiza en forma ramificada, mediante el uso del hipertexto y vínculos que favorece el acceso a la información y al aprendizaje colaborativo, permitiendo al sujeto interactuar con el medio en forma personal, y que 
en dicha interacción construya en forma significativa y adaptativa su conocimiento (Lara, 2004).

García Aretio (2002) menciona algunas ventajas de los materiales multimedia:

- $\quad$ El estudiante pasa a ser protagonista en la búsqueda de contenidos y en el descubrimiento de nuevos saberes.

- El sistema dispone de una gran capacidad de almacenamiento de información.

- Permite iniciar y desarrollar un diálogo, responder preguntas, resolver problemas, explorar, etc. Por lo que es elevado el control que tiene el estudiante.

- El estudiante puede controlar su propio progreso, la interactividad es total para reforzar el proceso.

- $\quad$ El proceso de enseñanza-aprendizaje se presenta individualizado, a la medida de las posibilidades, intereses y estilos de aprendizaje de cada uno.

- La calidad del proceso de enseñanza no varía nunca, siempre es constante y homogéneo en todos los momentos.

- El diseño suele ser atractivo, por lo que resulta fácil de usar y el aprendizaje resulta agradable al combinar diversos medios.

Los materiales con componente hipertextuales producen un aumento en el nivel de interactividad "mediante el hipertexto, el lector ha de ser activo al seleccionar su itinerario, al construir su propio texto, paralelo al del autor, por lo que las experiencias de leer y escribir textos cambian radicalmente" (García Aretio, 2002, p. 184). Los vínculos o enlaces proveen al alumno el acceso a la red no sólo para acceder a la información, mediante una exploración de sitios webs, sino también para establecer contacto con recursos que posibilitan el aprendizaje colaborativo con otro alumno o interactuar directamente con el tutor.

El diseño y desarrollo de estos materiales, mediante el empleo de múltiples medios en uno o varios soportes marcan un verdadero desafío, como afirma Piscitelli (2002, p. 17) "la proliferación de transmisores, medios, herramientas de difusión, etcétera, en vez de generar más sentido aumenta el ruido y la desinformación”, es así que, se presenta el planteo de pautas metodológicas de diseño basado en un marco teórico sistémico, que integre con coherencia y en forma integrada los distintos medios que constituyen un material interactivo. 


\section{LOS MATERIALES MULTIMEDIA DESDE UN ENFOQUE SISTÉMICO}

Desde una perspectiva sistémica, surgen nuevos conceptos y componentes a tener en cuenta en el estudio de estos materiales, así, un sistema se puede considerar como "un conjunto organizado de elementos diferenciados cuya interrelación e interacción supone una función global. Las relaciones se establecen entre las diversas partes y entre cada parte y el conjunto como totalidad" (Sánchez Cerezo, 1991, p. 480).

Por ello, la Teoría General de los Sistemas (T.G.S.) planteada por Bertalanffy no estudia a los sistemas a partir de sus elementos sino tratándolos a partir de su organización interna, sus interrelaciones, sus niveles jerárquicos, su capacidad de variación y adaptación, su conservación de identidad, su autonomía, las relaciones entre sus elementos, sus reglas de organización y crecimiento, su desorganización y destrucción. Una de las virtudes esenciales de la T.G.S. es la de tratar a los sistemas, sin prescindir de sus relaciones con su entorno, manteniendo además, las conexiones internas y externas de sus elementos. Todo lo cual no puede ser separado sin destruir la esencia del mismo, es decir, su unidad. Pues, una de las ideas básicas en T.G.S. es que el todo es más (y es otra cosa) que la suma de sus partes porque las características constitutivas de ese todo no es explicable a partir de las características de las partes aisladas.

Desde un punto de vista sistémico, un material interactivo educativo compatible con la corriente constructivista, debería tener las siguientes características:

- Ser un sistema abierto, es decir, un sistema real que interactúa con el entorno, le afecta sus influencias, pero a la vez, se diferencia de él, por tanto, es permeable a los estímulos externos.

- Tener una perspectiva holística: el todo es superior o diferente a las partes constitutivas.

- Ser recursivo: se muestra independiente y coherente, aunque se encuentre situado en el interior de otro sistema, o bien, aunque envuelva y contenga a otros sistemas.

- Ser sinérgico: un sistema no es la simple suma de efectos de cada una de sus partes, sino que la interrelación de sus componentes produce un efecto diferente y superior. 
- Ser un sistema realimentado: de acuerdo a las señales de entrada, el sistema por sí solo se reorienta o replantea continuamente su situación (principio de la cibernética) para poder a cumplir con su objetivo final.

Como se afirmó en el concepto de SIM educativo, la cualidad de la adaptación es clave para el funcionamiento de un material educativo, por tanto, se hace referencia implícita a sistemas abiertos. En estos sistemas, en la práctica sólo es significativo aquel entorno o ambiente a una parte del universo con el cual el sistema mantiene intercambios de cierta importancia y en forma frecuente.

Mediante un análisis termodinámico, y más precisamente de la entropía, será posible conocer la tendencia que lleva a cabo la evolución de estos materiales al interactuar como sistema abierto, ya que, en ese entorno se encuentra entre otros elementos la vinculación y la información que proporciona el usuario, de esta manera, un SIM se comportaría en forma adecuada y adaptada al perfil del usuario, según las características que presente las estimulaciones y flujos de información que provienen del medio.

Es así que, en la actualidad, las aplicaciones multimedia educativas, en su afán de favorecer el proceso de enseñanza aprendizaje, sólo lo puede conseguir desde el punto de vista de un "usuario estándar", del cual los programadores y diseñadores de la aplicación tomaron como modelo con argumentos dudosos y discutibles sobre lo que significa realmente el término "usuario estándar", sin considerar que cada individuo construye su conocimiento de diversas formas y proviene de conocimientos $\mathrm{y}$ acontecimientos previos diferentes.

Desde esta perspectiva, se debe tener en cuenta que la mayor parte de las aplicaciones informáticas con fines didácticos que existen en la actualidad, tienden a ser sistemas abiertos muy limitados, pues, los estímulos provenientes del exterior y sus realimentaciones, no influyen en forma determinante en cuanto a la esencia del funcionamiento y al desarrollo del mismo.

De esta manera, ante la presencia de innumerables estrategias y métodos que se utilizan para el desarrollo de materiales multimedia educativos, en donde el diseño no se encuadra bajo un claro sustento teórico o están enmarcadas en teorías prestadas que corresponden a la modalidad presencial o en determinadas reglas aisladas de diseño sin relación alguna entre sí, se introduce el concepto de entropía en la propuesta de desarrollo. 


\section{LOS PRINCIPIOS DE LA TERMODINÁMICA}

Desde los anales de la ciencia, la termodinámica presenta dos principios fundamentales:

- $\quad$ Primer principio de la termodinámica (ley de conservación de la energía): la energía no puede crearse ni destruirse, se transforma; el calor y el trabajo son mecanismos por los que los sistemas intercambian energía entre sí.

En cualquier máquina, hace falta cierta cantidad de energía para producir trabajo; es imposible que una máquina realice trabajo sin necesidad de energía. Una máquina hipotética de estas características se denomina "móvil perpetuo de primera especie". La ley de conservación de la energía descarta que se pueda inventar una máquina así. A veces, el primer principio se enuncia como la imposibilidad de la existencia de un móvil perpetuo de primera especie.

- Segundo principio de la termodinámica (concepto de entropía): la entropía se puede inferir como una medida de lo próximo o no que se halla un sistema al equilibrio; también se puede considerar como una medida del desorden (espacial y térmico) del sistema. Este principio, afirma que la entropía, o sea, el desorden, de un sistema aislado (donde el sistema no puede intercambiar ningún tipo de información con el ambiente) nunca puede decrecer. Por tanto, cuando un sistema aislado alcanza una configuración de máxima entropía, ya no puede experimentar cambios: ha alcanzado el equilibrio. La naturaleza parece, pues, "preferir" el desorden y el caos. Se puede demostrar que el segundo principio implica que, si no se realiza trabajo, es imposible transferir calor desde una región de temperatura más baja a una región de temperatura más alta.

El segundo principio impone una condición adicional a los procesos termodinámicos. No basta con que se conserve la energía y cumplan así el primer principio. Una máquina que realizara trabajo violando el segundo principio se denomina "móvil perpetuo de segunda especie", ya que podría obtener energía continuamente de un entorno frío para realizar trabajo en un entorno caliente sin coste alguno.

El concepto de entropía, tiene diversas interpretaciones, pues, pasa de ser una medida de la pérdida de calor, de energía (Clausius), a una medida de aleatoriedad o desorden (Boltzman) y a la vez está relacionada con la cantidad de información que se puede transmitir por un canal (Shannon y Weaver). 
Todos los sistemas, están sujetos al segundo principio, según el cual existe una magnitud llamada entropía, considerada como aquella energía que se disipa en forma espontánea como consecuencia de los procesos internos del sistema y que no podría volver a reutilizarse para producir trabajo.

En los sistemas cerrados o aislados, esta entropía aumenta progresivamente hasta llegar a lo que se conoce como equilibrio termodinámico, la máxima probabilidad es su progresiva desorganización y, finalmente, su fusión con el ambiente.

Los sistemas abiertos, al ser permeables con el medio, compensarían esa producción de entropía interna, incorporando materia, energía o información del ambiente, es decir, entropía negativa o neguentropía. La neguentropía es en sí una medida de orden; de este modo, mediante la extracción continua de orden u organización del medio, se mantiene el sistema estacionario y a un nivel alto de ordenamiento (nivel bajo de entropía). La información que recibe el sistema y que proviene del medio es la más importante corriente neguentrópica de que disponen los sistemas complejos, siempre considerando información toda aquella que es verdaderamente relevante para el sistema.

\section{Entropía en los sistemas cerrados}

Los sistemas cerrados, como muestra la Figura 1, son aquellos donde no tienen intercambio de información o energía con el entorno, todas las transformaciones implicadas quedan incluidas en ellos; tienen la tendencia natural al crecimiento de la entropía, es decir, la máxima probabilidad de los sistemas es su progresivo desorden y, finalmente, su homogeneización con el medio, pues, tienden irremediablemente a la desorganización total (muerte térmica o equilibrio termodinámico). El segundo principio de la termodinámica afirma que la entropía de un sistema cerrado nunca puede decrecer. Entonces, cuando un sistema de este tipo alcanza una configuración de máxima entropía, ya no puede experimentar cambios: ha alcanzado el equilibrio, y este cambio es irreversible (se da en un solo sentido natural, espontáneo y no se puede volver atrás). 


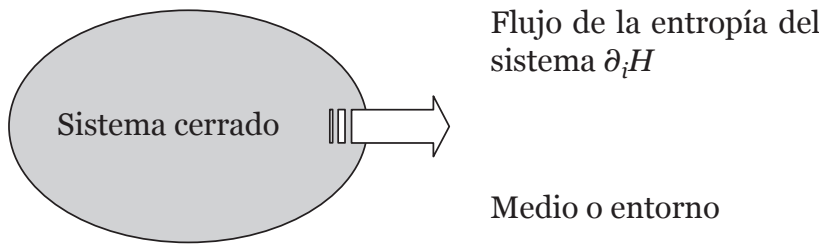

Figura 1. Entropía en un sistema cerrado

En lo que respecta a programas informáticos, se pueden considerar cerrados a aquellas programas herramientas tradicionales, como un procesador de texto, una planilla de cálculo, que sirven para desarrollar otros archivos documentos, estos programas herramientas, tiene diversos componentes de control y vías de comunicación restringidas con el medio que acentúan la fuerte diferencia entre el sistema y el medio (alto nivel de organización), el usuario bajo ningún punto de vista puede alterar el programa herramienta original, en estos casos el usuario (como un componente del medio o ambiente) se tiene que adaptar al sistema, lo que se lleva en la práctica mediante un estudio del funcionamiento de la herramienta. Cuanto más complejo es el estudio del uso de una herramienta informática, más rígido es su estructura interna. Teniendo, en consecuencia, una entropía muy baja, aunque el sistema se mantenga estático, sin evolucionar.

\section{La entropía en los sistemas abiertos}

En el mundo real, los sistemas son abiertos, un sistema abierto es aquel que importa información (corriente de entrada), la transforma (proceso de transformación) y luego exporta al ambiente esa nueva información. Con el producto de esa exportación, el sistema está en condiciones de obtener nuevamente sus corrientes de entrada necesarias para llevar a cabo el proceso de transformación, que lo caracteriza y diferencia del resto de los sistemas.

La realidad muestra como los organismos vivos como las estructuras artificiales creadas por el hombre presentan una tendencia a una mayor heterogeneidad (elementos del sistema bien diferenciados con respecto al medio con que interactúa) y niveles crecientes de organización. Ello es debido a que el incremento "natural" de la entropía que se produce en todo sistema es contrarrestado en los sistemas abiertos por la disipación de esta entropía con su entorno (flujo de entropía negativa), gracias precisamente a esos intercambios de flujos de información, cambios de objetivos o estructuras hacen que la entropía total del sistema pueda mantenerse constante, e incluso, disminuir; la Figura 2 evidencia los flujos involucrados. 


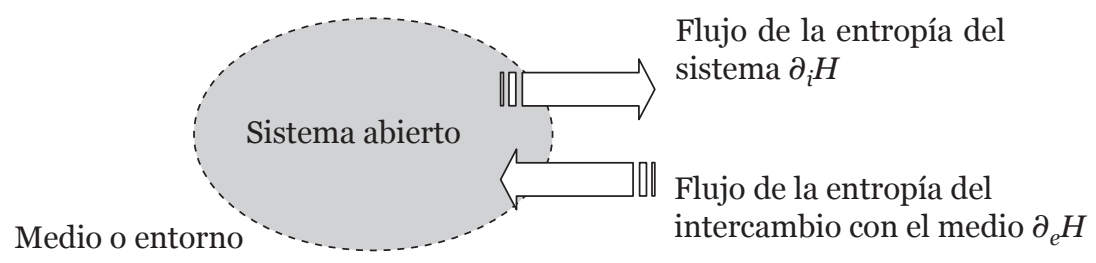

Figura 2. Entropía en un sistema abierto

Es evidente que estos flujos, que provienen del entorno producen perturbaciones e inestabilidades en el sistema, pero una asimilación adecuada de los mismos, y no su eliminación o neutralización, es la que permitirá que el sistema siga funcionando. De este modo, como afirma Sarabia (1995, p. 56) "en un sistema abierto, se deben tener en todo momento despejado sus canales de información con el entorno y asumir el conflicto que supone la aceptación de la diversidad, ya que en caso contrario, evolucionarán como los sistemas cerrados alcanzando su particular muerte térmica y marcándose como fin una degradación del fin original". Por tanto, permeabilizar la frontera sistema-entorno llevará al sistema a una situación de inestabilidad, pero si el sistema tiene un diseño adecuado, lo conducirá a un nivel más evolucionado y complejo.

Se pueden mencionar como ejemplos de sistemas abiertos en informática a aquellos archivos derivados de programas herramientas o de algún lenguaje de programación como documentos o archivos fuentes, así, los mencionados archivos pueden ser modificados por el usuario (dentro de ciertos códigos impuestos por el programa herramienta o lenguaje de programación).

Una entropía creciente positiva implica la idea de un proceso espontáneo e irreversible; en termodinámica, un procedimiento irreversible a aquel proceso natural, que tiene lugar sin ninguna influencia externa y que disipa energía; que es el caso cuando el calor pasa de un cuerpo más caliente a un cuerpo más frío si están en contacto, o la expansión libre de un gas. En un proceso irreversible no se puede volver a una etapa anterior ya que éstas presentan un alto grado de inestabilidad. Los cambios espontáneos siempre van acompañados de una reducción en la "calidad" del flujo de la información, energía o materia, en el sentido de que es degradada a una forma más dispersa y caótica (Atkins, 1991).

Una entropía constante, en cambio, hace referencia a un proceso "antinatural" y reversible, el proceso reversible "es aquel que se puede ejecutar en ambas direcciones, de manera que todos los cambios que ocurran en cualquier parte del 
procedimiento directo se revierten exactamente en las etapas correspondientes del proceso inverso" (Glasstone, 1972, p. 180). Los procesos reversibles son cambios compensados, donde están involucrados agentes externos, encontrándose el sistema en equilibrio con sus alrededores en cada etapa del proceso, sin degradación de la calidad de la energía, sin dispersarla caóticamente (Atkins, 1991).

En el desarrollo de materiales interactivos educativos se debe considerar que su interacción con el entorno, lleve al sistema a un nivel más o menos controlable de organización, lo que se hace lógico considerando un material que comprenderá contenidos, actividades y trayectos didácticos, en consecuencia, la entropía debe mantenerse en un valor constante, de esta manera, la cantidad de información que adquiere el sistema se verá jerarquizada (el sistema habrá evolucionado a un estado superior de organización).

Por tanto, se busca considerar al material interactivo educativo como un sistema permeable con una estructura básica que, para que vaya desarrollándose, precisará sustentarse de señales proveniente del entorno, donde le proveerá de información adicional para neutralizar la variación de la entropía del sistema, de esta forma irá expandiéndose en forma ordenada en el desarrollo de módulos que se generarán de acuerdo a los requerimientos que solicite el medio (perfil del alumno + hardware).

La razón por la que se incluye estos elementos, es que se busca la evolución del software por la acumulación seleccionada de información, proveniente de datos del entorno (principalmente proporcionada por la interacción con el alumno); proveyendo el sistema como respuesta, tanto de actividades como de contenidos adecuadas a esas interacciones.

\section{LA ENTROPÍA Y LA CANTIDAD DE INFORMACIÓN}

El proceso de enseñanza-aprendizaje lleva implícito un proceso de comunicación, Marqués (1995) describe un modelo de comunicación, específicamente para software educativo donde implica la existencia de un receptor, que recibe un mensaje codificado transmitido por un emisor a través de un canal y en un ambiente determinado:

- El emisor o fuente: es quien decide lo que hará el programa, en este caso está representado por el diseñador o autor del programa.

- $\quad$ El mensaje: es el material interactivo que se utiliza, sus contenidos. Representa la dimensión semántica del material. 
- El canal: es el medio a través del cual se transmite el mensaje. En este caso, el ordenador, que es capaz de leer el soporte (magnético u óptico) que contiene el programa.

- El código o lenguaje: son los símbolos y las reglas con las que se transmite el mensaje, es decir, su forma de organización (las pantallas y el entorno de comunicación del programa en general, con su interfaz). Representa la dimensión estructural-sintáctica del software educativo.

- $\quad$ El contexto, medio o ambiente: se refiere al entorno físico y social donde se usa el programa: el lugar, el momento dentro del desarrollo curricular, la organización, representa la dimensión pragmática.

- El destinatario o receptor: es el alumno que interactúa con el material.

Cabe aclarar, que en un material interactivo, dado su carácter de intercambio de flujo bidireccional, en cualquier momento se pueden suplantar los roles del emisor y receptor en el proceso de comunicación. Por ende, se puede considerar como un proceso de comunicación unidireccional en el cual se intercambian en forma alternativa el rol de emisor y receptor. Para García Mayoraz (1989) el mensaje, como parte constitutiva de la comunicación, es la forma en que la fuente suministra información, para lo cual, se vale de un lenguaje absolutamente convencional y arbitrario constituido por una serie de símbolos y se dirige a un receptor que, para interpretarlo, tendrá en primer lugar que decodificar esos símbolos y sus relaciones utilizando el mismo código. El mensaje circulará por un medio físico que es el canal.

En la Figura 3 se presenta un modelo simple del proceso de comunicación:
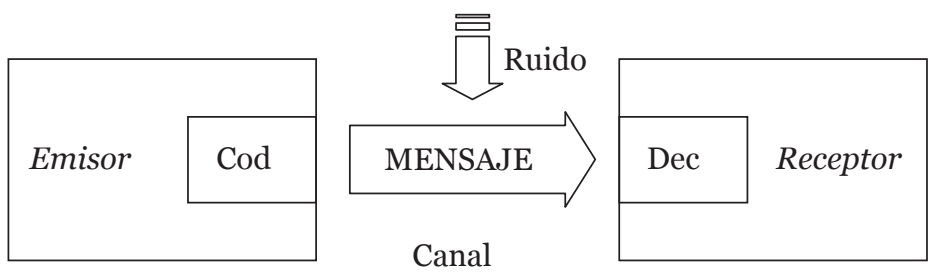

Figura 3. Proceso de comunicación unidireccional

Si los mensajes posibles son muchos, entonces la entropía es máxima cuando son iguales las probabilidades de elegir (o recibir) cualquier mensaje, en otras palabras, cuando existe la mayor libertad posible en optar entre los diversos mensajes disponibles, sin que se ejerza influencia alguna en dicha elección. 
Shannon y Weaver (1949) plantearon el concepto de cantidad de información $I$ que aporta el símbolo $S_{i}$ en función a la probabilidad de su aparición $p$ como:

$$
\mathrm{H}=\sum \mathrm{p}_{\mathrm{i}} \cdot \mathrm{I}\left(\mathrm{s}_{\mathrm{i}}\right)=-\sum \mathrm{p}_{\mathrm{i}} \mathrm{I}\left(\mathrm{s}_{\mathrm{i}}\right)
$$

Donde:

$p_{i}$ : es la probabilidad que se cumpla el suceso o el mensaje $s_{i}$ un número entre o (un evento improbable) y 1 (un evento posible).

$s_{i}$ : símbolos que tiene la fuente $\left(s_{1}, s_{2}, \ldots, s_{n}\right)$

El término cantidad no se refiere a la cuantía de datos, sino a la probabilidad de que un mensaje, dentro de un conjunto de mensajes posibles, sea recibido.

A la entropía $H$, Shannon la definió como la cantidad de información promedio de estos mensajes, definida de la siguiente forma:

$$
\mathrm{H}=\sum \mathrm{p}_{\mathrm{i}} \cdot \mathrm{I}\left(\mathrm{s}_{\mathrm{i}}\right)=-\sum \mathrm{p}_{\mathrm{i}} \cdot \log _{2}\left(\mathrm{p}_{\mathrm{i}}\right)
$$

Un caso particular es aquella gráfica, como lo demuestra la Figura 4, que representa la información y la entropía proporcionada por una fuente binaria (sólo puede tomar dos valores, sucesos o eventos), en este caso, la función de entropía tendrá la siguiente expresión:

Partiendo de la expresión general: $\mathrm{H}=-\mathrm{p}_{1} \log _{2} \mathrm{p}_{1}-\mathrm{p}_{2} \log _{2} \mathrm{p}_{2}$

También se tiene la siguiente relación: $\mathrm{p}_{1}+\mathrm{p}_{2}=1 \Rightarrow \mathrm{p}_{2}=1-\mathrm{p}_{1}$

Entonces, $\mathrm{H}=-\mathrm{p} \log _{2} \mathrm{p}-(1-\mathrm{p}) \log _{2}(1-\mathrm{p})$ 


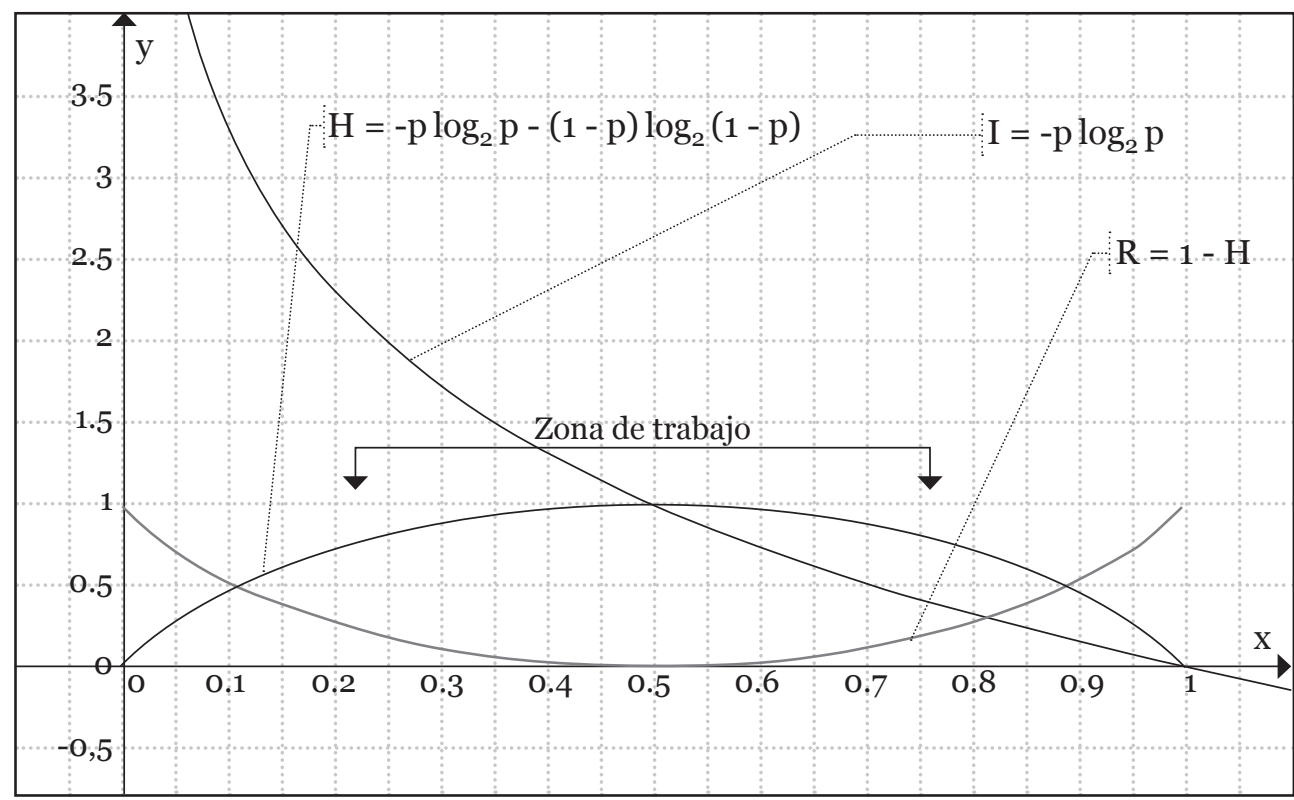

Figura 4. Entropía y cantidad de información para una fuente binaria

Considerando las coordenadas que cumplen con la condición de entropía de la fuente $H=O$ se puede inferir que:

- Cuando no hay una probabilidad de que ocurra un evento determinado, entonces la elección de un símbolo determinado es nula $(p=o)$, implica que la cantidad de información recibida $I$ será extremadamente alta (tiende al infinito, hay una gran noticia).

- Cuando se cumpla el suceso más probable $(p=1)$, la cantidad de información $I$ vale cero, no hay noticia, sólo redundancia (una mayor claridad y significado en el mensaje a costa de una información transmitida nula).

En estos dos puntos el mensaje carece de sentido, ya sea porque aporta demasiada información en un extremo, el mensaje es imposible que sea proporcionado por su bajo nivel de significado o porque el mismo es totalmente redundante en el otro extremo, siempre se da el mismo mensaje.

Por otro lado, estudiando la curva de $H$, se concluye que la condición $H=H_{M A X}$ se manifiesta cuando la información de todos los símbolos son equiprobables, 
es decir, todos los sucesos tienen la misma posibilidad de ser recibidos, hay una gran diversidad en cuanto a la selección de cada símbolo, no hay redundancia en el mensaje, por tanto, la cantidad de información $I$ recibida es alta e igual para todos los eventos que puedan suceder, justamente por esta causa, todo el mensaje es información efectiva. En la práctica no existen canales perfectos por donde no pueda ingresar el ruido, es así que, para combatir el ruido es necesario emplear la redundancia.

Cuenca (1999) destaca que la información no es sinónimo de significado. El término información no se refiere tanto a lo que se dice, sino a lo que se podría decir. Por tanto, la información es la medida de la libre elección del mensaje. Cuando alguien se enfrenta a la situación de tener que elegir entre dos mensajes alternativos, se dice que la información asociada a esta situación es la unidad. El concepto de información se refiere, no a los mensajes individuales sino, a la totalidad. Que la información se mida por la entropía es natural, si se piensa que, la información se asocia al grado de libertad de elección que se tiene al construir los mensajes. Dada una fuente de información, se puede decir que ésta situación está muy organizada y no se caracteriza por un elevado grado de azar o de elección, es decir, la información (o la entropía) es baja.

De esta manera, aquellos materiales didácticos rígidos, donde no hayan una gran cantidad de mensajes para seleccionar tendrán una entropía muy baja, en caso de que no existan posibilidades de seleccionar diversos eventos que constituye el mensaje, o siempre se selecciona el mismo, estaremos ubicados en los extremos de la curva (izquierdo o derecho, respectivamente).

En consecuencia, considerando que se prefiere un estadio medio entre la organización y la cantidad de información suministrada, aunque haya una gran cantidad de mensajes para seleccionar, se propone la zona de trabajo cercano al valor máximo de entropía $\left(H \approx H_{M A X}\right)$.

Al considerar la relación que existe entre la información y el significado, Eco (1979) es concluyente al resumir estas condiciones:

- Cuanto mayor es la cantidad de información, tanto más difícil es comunicarla de algún modo; cuanto más claramente se comunica un mensaje, menos información se transmite. 
- El aumento de significado en la transmisión del mensaje supone pérdida de la cantidad de información y el aumento de la información supone pérdida de significado.

En el intercambio de información se debe considerar la realimentación, la misma puede ser negativa (o de compensación) o positiva (o de refuerzo).

La realimentación negativa es la que mantiene un nivel organizado del sistema, lo estabiliza y lo lleva a cumplir con la misión que tiene, es decir, toda información valiosa para que el sistema se mantenga lejos del equilibrio u homogeneización con el ambiente.

En cambio, la realimentación positiva tiende a amplificar los efectos de la condición inicial y pueden transportar al sistema a nuevos estados. En comunicación, la realimentación es una señal en la cual se reingresa información al sistema mediante algún periférico de entrada, por ejemplo, utilizando el teclado o el mouse en equipos informáticos, que indica si el mensaje se ha recibido e interpretado correctamente.

Otra forma de hacer crecer el valor de $H$ es aumentando el número de mensajes; concretamente, cuantas más alternativas haya, mayor será $H$ para probabilidades aproximadamente iguales.

Se puede comparar la entropía (o libertad de elección) de cierta fuente de información con el valor máximo que esta entropía podría alcanzar si estuviese sujeta sólo a la condición de que la fuente continúe empleando los mismos símbolos. Se llama entropía relativa $H_{r}$ de una fuente a la relación existente entre la entropía real y la máxima:

$$
\mathrm{H}_{\mathrm{r}}=\frac{\mathrm{H}}{\mathrm{H}_{\mathrm{MAX}}}
$$

El complementario de la entropía relativa se llama redundancia $R$ y es la fracción del mensaje que no está determinada por la libre elección del emisor:

$$
\mathrm{R}=1-\mathrm{H}_{\mathrm{r}}=1-\frac{\mathrm{H}}{\mathrm{H}_{\mathrm{MAX}}}
$$

El concepto de redundancia corresponde al sentido común que se tiene de esta palabra, se interpreta como aquella fracción del mensaje innecesaria (y por tanto, repetitiva) ya que si faltara en el mensaje, éste seguiría completo, o podría completarse y ser entendible. 
Para García Mayoraz (1989, p. 35) “la información es eminentemente entrópica, repele la redundancia, en cambio la comunicación en su núcleo informador de su contenido tiene que ser información, pero en su caparazón, su soporte, tiene que ser redundancia". De esta manera, surgen dos tipos de información que transmite el sistema, una referida a la consecución de los objetivos del material instructivo (contenidos y actividades) y otra que transmite el soporte o dispositivo de almacenamiento. Es evidente que la información relacionada con el soporte debe tener una alta redundancia, para que el empleo del material sea transparente y se pueda acceder fácilmente a los contenidos.

La teoría de la comunicación introduce, por un lado, el concepto de ruido (que hace referencia a las perturbaciones en la transmisión de la información), asociado con la noción de la entropía positiva de la $2^{\text {a }}$ ley de la termodinámica, ya que disminuye la integridad de la comunicación y produce desorden en los mensajes; y por otro lado, el concepto de redundancia (repetición de elementos dentro de un mensaje) que trata de evitar el fracaso en la transmisión de la información y se puede relacionar con la entropía negativa, favoreciendo la comunicación efectiva.

García Mayoraz (1989, p. 197) enfatiza que "el mensaje debe ser claro, el lenguaje transparente, los signos preponderantemente transparentes”. La buena utilización del código asegura la correcta recepción del mensaje, su completa inteligibilidad, la ausencia de todo ruido, porque el lenguaje es precisamente un filtro que sólo permite pasar aquellos significados que están ordenados en el mismo código; el lenguaje impone el orden y el control. El código está ordenado en sus elementos y en sus relaciones; con él se puede hacer un mensaje bien claro, la entropía propia del código, entonces, es muy baja. Sin embargo, para aumentar la cantidad de información, se tendrá que aumentar la entropía, introduciendo desorden en el código, es decir ruido en el mensaje, para que el sistema evolucione a un orden superior. Entonces, ruido (relacionado con el aumento de la información) y redundancia (congruente con la claridad en que se emite un mensaje) compensan la entropía para que se mantenga en un valor más o menos constante. En realidad, no se propone trabajar con un valor alto o bajo de entropía, sino que la misma permanezca más o menos constante, lo que implica que es relevante el intercambio de información del sistema con el medio.

\section{Desplazamiento de la entropía en sistemas cerrados}

En lo referido a materiales multimedia educativos, aquellos softwares que sólo suministran información sin recabar información del ambiente (perfil del usuario y del hardware, por ejemplo) tienen al principio una entropía alta, proveniente de 
la novedad de los contenidos, pero luego, a causa de que los mismos con el tiempo dejan de ser novedosos, comienzan a desplazarse hacia la derecha, como queda evidente en la Figura 5, el sistema se vuelve cada vez más predecible y redundante para el usuario.

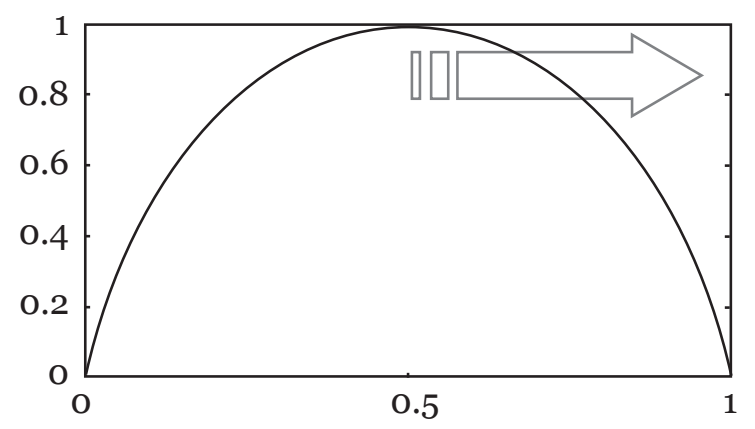

Figura 5. Material multimedia cerrado

\section{Desplazamiento de la entropía en sistemas abiertos}

En el caso particular del SIM educativo propuesto, la tendencia a la disminución de la entropía (como sucede en los sistemas cerrados) es contrarrestada por la información que recaba el sistema de su medio, mediante las realimentaciones y de los componentes azarosos, lo que hace que la variación de la entropía se mantenga más o menos constante, como muestra la Figura 6.

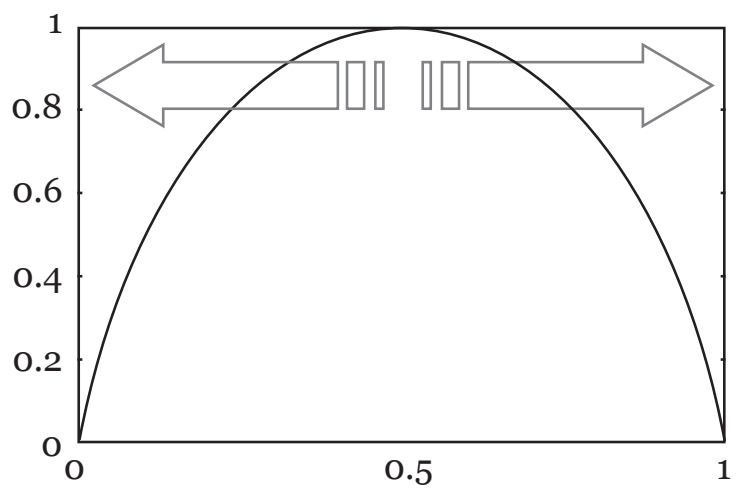

Figura 6. Material multimedia abierto

Cabe aclarar que esta neutralización de entropías no será permanente, pues, el flujo entrópico hacia la derecha prevalecerá indefectiblemente a medida que se vayan 
cumpliendo los objetivos o metas de aprendizaje que tiene el material interactivo, hasta llegar a un punto en que dicho material habrá cumplido con sus objetivos y la entropía tenderá naturalmente a cero.

Se debe considerar que la entropía desde el punto de vista cuantitativo no será igual para todos los usuarios que interactúan con el material interactivo, dependerá de varios factores, como la adaptabilidad del usuario al medio, conocimientos, motivaciones y experiencias previas que tengan que ver con la temática abordada por el material, etc. Entonces, la velocidad de desplazamiento de la entropía variará según el perfil del alumno que interactúa con el material.

\section{EL DISEÑO DE PROGRAMAS VOLÁTILES Y LAS PERTURBACIONES IMPLICADAS}

Desde el punto de vista didáctico, lo relevante del enfoque constructivista es que el alumno tiene el control del proceso cuando puede tomar decisiones, de acuerdo a su propio estado cognitivo y de sus propias necesidades. Squires (1999) presenta una paradoja considerando el diseño de programas multimedia educativos desde una perspectiva constructivista: si, al intentar desarrollar ambientes de aprendizaje eficaces estamos al mismo tiempo reprimiendo los niveles de libertad (disminuyendo la entropía) para que los mismos aprendices puedan tomar decisiones sobre su propio aprendizaje.

De acuerdo a las intenciones de diseño que se tuvo a realizar un programa multimedia, los usuarios pueden modificar el diseño original del material para satisfacer sus propias necesidades, dependiendo de los objetivos que se persiguen y el grado de delegación que permite el software. Es decir, de la manera en que tutores y alumnos usan el material, pueden reformar las intenciones originales que tuvo el diseñador. Desde la perspectiva del diseño Squires la llama "perturbación delegada".

Esta delegación puede presentarse en tres niveles, dependiendo si el software incorpora intenciones del diseño educativo explícitas, implícitas o ausentes. El uso alterado del material en la primera categoría requerirá un esfuerzo consciente por frustrar las intenciones de los diseñadores. En el segundo caso, el software puede usarse subversivamente sin que el usuario esté consciente de él. En el tercer caso, por definición, una ausencia de apuntalar intenciones del diseño educativo, a menudo debido a que el software originalmente fue pensado para uso general, por lo que 
es "secuestrado" para los propósitos educativos, en este caso, se pone la carga en el maestro y/o aprendiz para modificar las intenciones no-educativas del diseñador.

En cuanto a "la perturbación incorporada", consiste en dar énfasis al papel activo y determinante de los alumnos en configurar sus propios ambientes de aprendizaje para resonar de acuerdo a sus propias necesidades, haciendo eco de las nociones de aprendizaje con tecnología a través del "compromiso atento" y los aprendices como diseñadores intentando representar su propio conocimiento, de esta manera, se busca la significancia personal.

La alteración incorporada lleva a diseños del software que son volátiles por naturaleza, respondiendo al cambio y necesidades idiosincrásicas de aprendices. Un rasgo crucial de subversión incorporada es la creación de un enlace entre la exploración y expresión proporcionando una realimentación intrínseca que representa los efectos de las acciones de los alumnos.

En consecuencia, una síntesis de los rasgos esenciales de una visión constructivista del aprendizaje en el desarrollo de materiales multimedia educativos es que debe iluminar las características de la perturbación delegada y mantener pautas de diseño de aplicaciones arteras que ofrecen la perturbación incorporada.

Así, los programas deben ser volátiles con respecto a su diseño y perturbables en cuanto a su utilización. La idea de volatilidad está proporcionando de alguna manera, la idea del grado de permeabilidad del sistema abierto con respecto a la estimulación del entorno, $y$, también por la reversibilidad que caracteriza a los procesos involucrados. Lo subversivo, con el nivel de maleabilidad (grados de libertad, entropía) que pueden presentar, mediante el proceso adecuado del tránsito de información tanto interna como aquella proveniente del entorno que llevará a cabo el software.

Los programas actuales de computación referidos a usuarios finales presentan secuencias preestablecidas de acciones, son "secuencias de instrucciones escritas en un lenguaje determinado y que vienen a representar la solución a un problema" (Sánchez Cerezo, 1991, p. 429), no obstante, para enfrentar la incertidumbre de la acción que provoca, en nuestro caso, la interacción del sistema con el medio, es necesario el recurso de la estrategia, que permite a partir de una decisión inicial, imaginar un cierto número de escenarios para la acción, escenarios que podrán ser modificados según las informaciones que lleguen al sistema en el curso de la acción y según los elementos aleatorios que sobrevendrán y perturbarán esa acción. 
Es, entonces, donde se vislumbra una arquitectura modular para los SIM, cuyo conjunto de módulos interconectados constituyen una red de estados intercambiables y reversibles.

Morin (1999) expresa que la estrategia debe prevalecer sobre el programa, ya que éste al presentar secuencias determinadas de antemano en un entorno estable, al ocurrir alguna alteración en las condiciones externas, se bloquea. En cambio, la estrategia elabora un espacio de acción examinando las certezas e incertidumbres de la situación (interacciones del usuario y el hardware), las probabilidades y las improbabilidades, se pueden plantear estrategias utilizando secuencias cortas de programas. Pero en un entorno inestable e incierto, como el que presenta un proceso enseñanza-aprendizaje, debe imponerse la estrategia.

Si se considera que el proceso de enseñanza y aprendizaje es complejo y que cada individuo aprende de distinta manera, las aseveraciones de Morin desde una perspectiva general, deben ser incluidas en el ámbito del diseño de los programas multimedia educativos, entonces, para que el proceso pueda ser efectivo y eficaz, los mencionados programas que existen en la actualidad deben dejar de ser programados para elevarse (o descender, según el punto de vista) a otra condición, la de ser un medio que no sólo almacene y presente información; sino que realmente pueda dar las herramientas necesarias para formar al individuo, planteando diversas estrategias de acuerdo a señales realimentadas implicadas en el proceso de enseñanza-aprendizaje.

\section{CONCLUSIONES}

Se puede concluir que ciertos conceptos termodinámicos como la entropía $\mathrm{H}$ pueden ser útiles en el planteo de diseño de materiales multimedia educativos, de esta manera, teniendo en cuenta las curvas para dos eventos (Figura 4): la condición $H=H_{M A X}$ es ideal, pues, el diseñador de materiales siempre tendrá un guión, y así, el control que puede ejercer el alumno nunca será total. La posición de los puntos extremos $H=0$ proporciona un mensaje sin significado. La entropía $H$ aumentará con el incremento de símbolos, lo que implicará un mayor grado de libertad para que el alumno pueda realizar su propio trayecto, sin embargo, se incrementará la complejidad, volviéndose caótica, de esta manera, será necesario agregar elementos de control y regulación que harán que la entropía se mantenga estable. El grado de interactividad proporcionado al alumno está dado por diversos caminos de información que provee el material, las realimentaciones. 
La zona de trabajo propuesta a entropía constante implica una mayor libertad para el alumno, pues, puede elegir la opción simbólica que más le sea afín, de acuerdo a sus conocimientos previos, experiencias, motivaciones y estilo de aprendizaje, así, como sistema abierto, tendrá la posibilidad de usar el material como él prefiera y con las características que quiera (podrá ser una enciclopedia, un tutorial, etc.).

La propuesta teórica de un SIM, tiene como objeto sistémico, incorporar componentes aleatorios, relacionado con el ruido, dicho elemento tiene la función de neutralizar la entropía naturalmente baja que producen las aplicaciones producidas por los ordenadores y la redundancia que esto acarrea, de esta manera, con la presencia al azar de diversos eventos o símbolos se contempla trasladar la función de entropía hacia un valor controlable, haciendo que estos sucesos sean realmente novedosos para el alumno que interactúa con el material. Sin embargo, en la práctica, las herramientas de autor que existen limitan desde su propia concepción, la apertura de las fronteras de los materiales interactivos desde el punto de vista sistémico.

De esta manera, respaldamos el desarrollo de un nuevo material instructivo dinámico, con una entropía constante, que va más allá de lo intangible, una aplicación volátil, modular, formando un todo tridimensional, a la medida de las necesidades del alumno, sin limitaciones y adaptable al usuario (perturbables). Cambiando el rol de alumno pasivo a un alumno protagonista de su propio proceso, constituyéndose también en el diseñador de su propio material.

\section{REFERENCIAS BIBLIOGRÁFICAS}

Atkins, P. (1991). Fisicoquímica. Wilmington: Addison-Wesley Iberoamericana.

Castellan, G. (1987). Fisicoquímica. Wilmington: Addison-Wesley Iberoamericana.

Cuenca, J. L. (1999). Tres lugares comunes para la entropía. Revista Encuentros Multidisciplinares. [en línea] Disponible en: http://www. encuentros-multidisciplinares.org/ Revistan\%C2\%BA3/N\%C2\%BA\%203\%2 oJos\%C3\%A9\%20Luis\%20Cuenca\%20T adeo.htm [consulta 2007, 2 de marzo].

Eco, U. (1979). Obra abierta. Barcelona: Ariel.
García Aretio, L. (2002). La educación a distancia. De la Teoría a la Práctica. Barcelona: Ariel.

García Mayoraz, J. E. (1989). Entropía/ Lenguajes. Buenos Aires: Hachette.

Glasstone, S. (1972). Tratado de química física. Madrid: Aguilar.

Lara, L. R. (2004). Introducción a un modelo complejo de software multimedia educativo. [en línea] Disponible en http://www.um.es/ead/red/12/lara.pdf [consulta 2007, 15 de abril].

Marqués, P. (1995). Software educativo. Barcelona: EMA-Estudis.

Morin, E. (1999). La cabeza bien puesta. Buenos Aires: Nueva Visión. 
Morin, E. (2001). Los siete saberes necesarios para la educación del futuro. Buenos Aires: Nueva Visión.

Padula Perkins, J. (2002). Una introducción a la educación a distancia. Buenos Aires: Fondo de Cultura Económica.

Piscitelli, A. (2002). Ciberculturas 2.o. Buenos Aires: Paidós.

Sánchez Cerezo, S. (1991). Léxicos tecnología de la educación. Madrid: Santillana.

Sarabia, A. (1995). La teoría general de sistemas. Madrid: Isdefe.
Shannon, C.; Weaver, W. (1949). The mathematical theory of communication. Illinois: University of Illinois Press.

Squires, D. (1999). Educational software and learning: subversive use and volatile design. IEEE Proceedings of the $32^{\text {nd }}$ International Conference on System Sciences, Hawaii.[enlínea]Disponibleen: http://www.computer.org/proceedings/ hicss/0001/00011/00011079.PDF [consulta 2004, 6 de agosto].

\title{
PERFIL ACADÉMICO Y PROFESIONAL DEL AUTOR
}

Luis Rodolfo Lara. Master en Tecnología Educativa (Universidad de Salamanca, España), Diplomado Universitario en Diseño y Gestión de Proyectos de E-learning y Educación a Distancia (Universidad Nacional de San Martín, Argentina), Ingeniero Electricista (Universidad Nacional de Tucumán, Argentina), Director del proyecto de investigación: "Análisis de sistemas interactivos multimedia educativos". Profesor de la cátedra "Fundamentos de educación a distancia", Facultad de Ciencias Exactas y Naturales, Universidad Nacional de Catamarca. E-mail: reolara@educ.ar

Web: http://www.educomdigital.com.ar/sim

DIRECCIÓN DEL AUTOR:

\author{
FaCEN, UNCa, \\ Av. Belgrano 300, (4700) \\ San Fernando del Valle de Catamarca, \\ Catamarca, Argentina
}

Fecha de recepción del artículo: 04/09/07

Fecha de aceptación del artículo: 11/03/08 